\title{
Imaging sparse metallic cylinders through a local shape function Bayesian compressive sensing approach
}

\author{
Lorenzo Poli, Giacomo Oliveri, and Andrea Massa* \\ ELEDIA Research Center, Department of Information Engineering and Computer Science, \\ University of Trento, via Sommarive 5, 38123 Trento, Italy \\ *Corresponding author: andrea.massa@ing.unitn.it
}

Received February 19, 2013; accepted May 5, 2013;

posted May 9, 2013 (Doc. ID 185506); published May 28, 2013

\begin{abstract}
An innovative method for the localization of multiple sparse metallic targets is proposed. Starting from the local shape function (LSF) formulation of the inverse scattering problem and exploiting the multitask Bayesian compressive sensing (MT-BCS) paradigm, a two-step approach is described where, after a first estimation of the LSF scattering amplitudes, the reconstruction of the metallic objects is yielded through a thresholding and voting step. Selected numerical examples are presented to analyze the accuracy, the robustness, and the computational efficiency of the LSF-MT-BCS technique. (c) 2013 Optical Society of America
\end{abstract}

OCIS codes: (100.3190) Inverse problems; (100.6950) Tomographic image processing; (280.0280) Remote

sensing and sensors; (290.3200) Inverse scattering; (350.4010) Microwaves.

http://dx.doi.org/10.1364/JOSAA.30.001261

\section{INTRODUCTION}

Locating and shaping perfect electric conductors (PECs) illuminated by known electromagnetic waves [1-ㅗ] is a key problem in many applications such as geophysical prospecting [5], localization of buried metallic cylinders []], detection of tunnels and pipelines [7], and retrieval of surface profiles [8]. In such a framework, the development of inversion strategies specifically devoted to image "sparse" metallic objects (sometimes referred to as thin [9]) embedded in large investigation domains has recently gathered great attention because of its complexity [9] as well as occurrence in fault detection of metallic grids [10], biomedical monitoring [11], far and near source localization $[\underline{12}, \underline{13}]$, nondestructive testing [14,15], and ground-penetrating radar [16-18]. While several techniques exploit global optimization strategies [19-21] (e.g., tabu search [22], genetic algorithms [6,7], differential evolution [23], and particle swarm optimizers [24]), the use of singular value decomposition-based methods $[4,25]$ and level set techniques [26] has been considered, as well. However, only few methodologies, generally based on approximate formulations (e.g., physical optics), have dealt with sparse scatterers [9,27]. As a matter of fact, most inversion techniques for PEC objects model the unknown contour as a function of the polar angle $[6,7,21,24,28,29]$ and usually require the approximate knowledge of the object centers [30] or the number of objects $[31,32]$. Because of these assumptions, they do not efficiently apply to the retrieval of multiple sparse scatterers. Alternative formulations, based on the local shape function (LSF) technique [30,33-36], seem to be more suitable candidates for such a purpose. Indeed, the LSF method employs a cell-based discretization of the investigation domain, enabling the effective representation of sparse scatterers as the superposition of (a few) thin metallic wires. On the other hand, the arising inverse problem turns out to be strongly nonlinear because of the multiple reflections in the wire model [30,34,35]. Recently, some countermeasures to this latter issue have been proposed [37], but they suffer from nonuniqueness because of the presence of nonradiating currents; then suitable regularization techniques (e.g., Tikhonov [37]) are required. Moreover, the information coming from different illuminations is a posteriori combined, resulting in a suboptimal exploitation of the available physical information [37].

In this paper, the new technique proposed for the localization of sparse metallic scatterers is aimed at (i) modeling thin objects through the LSF approach, (ii) regularizing the inverse problem at hand by means of a sparsity-based constraints, and (iii) combining in the inversion the information collected at different views. More specifically, the available physical a priori knowledge [(ii) and (iii)] is managed in a probabilistic fashion by means of a multitask Bayesian compressive sensing (MTBCS) [38] formulation because of the following considerations:

- Compressive sensing (CS) strategies are natural choices for the solution of ill-posed inverse scattering problems whenever an a priori knowledge of the sparseness of the unknowns is available [39];

- CS methodologies are also numerically efficient for highdimension problems [40,41];

- Unlike deterministic CS methods directly enforcing sparseness constraints by adding a sparsity term (e.g., the weighted $\ell_{1}$-norm) in the cost function, BCS techniques do not require the problem kernel to satisfy specific properties such as the restricted isometry property [41,42];

- MT-BCS approaches efficiently take into account mutual relations among correlated informations from different illuminations of the investigation domain $[38,43]$ thanks to the multitask formulation;

- The effectiveness and the efficiency of BCS-based techniques have already been proved in several electromagnetic problems (e.g., inverse problems [42-45] and array synthesis or diagnostics [46-49]). 
The outline of the paper is as follows. After describing the LSF framework of sparse inverse scattering problems (Section 2), the probabilistic formulation of the imaging strategy is introduced by focusing on the application of the multitask CS technique (Section 3). Representative numerical results are then provided to validate the proposed approach as well as to investigate its features and potentialities (Section 4). Some conclusions eventually follow (Section 5).

\section{SPARSE LSF PROBLEM FORMULATION}

Let us consider a two-dimensional investigation domain of extension $\Omega$ comprising a set of sparse PECs lying in a (also nonconnected) region $\Omega_{\mathrm{PEC}}\left(\Omega_{\mathrm{PEC}} \subset \Omega\right)$ and illuminated by $V$ known time-harmonic transverse-magnetic plane waves impinging from the angular directions $\theta^{v}, v=1, \ldots, V$ whose associated incident fields are $f^{v}(\mathbf{r}) \hat{\mathbf{z}}$ (for the sake of brevity, the time-dependency factor $\exp (-j \omega t)$ is implied and omitted hereinafter), $v=1, \ldots, V$, being $\mathbf{r}=(x, y)$, and $z$ is the axis of the cylindrical geometry.

By applying the LSF method [30], $\Omega$ is discretized into a set of uniform cells of area $\delta$ and centered at $\mathbf{r}_{n}, n=1, \ldots, N$. Moreover, a binary LSF, $\gamma \triangleq\left\{\gamma_{n}, n=1, \ldots, N\right\}$, is associated with the cell grid such that $\gamma_{n}=1$ if a metallic object occupies the $n$th cell and $\gamma_{n}=0$ otherwise:

$$
\gamma_{n}=\left\{\begin{array}{ll}
1, & \mathbf{r}_{n} \in \Omega_{\mathrm{PEC}} \\
0, & \mathbf{r}_{n} \notin \Omega_{\mathrm{PEC}}
\end{array} \quad n=1, \ldots, N\right.
$$

It is worth noting that this is equivalent to replacing the metallic region $\Omega_{\mathrm{PEC}}$ with $S \triangleq\|\gamma\|_{\ell_{0}}$ thin wires if $S \ll N$ (i.e., $\gamma$ turns out to be a sparse vector).

The scattered electric field, $e^{v}(\mathbf{r}) \hat{\mathbf{z}} \triangleq t^{v}(\mathbf{r}) \hat{\mathbf{z}}-f^{v}(\mathbf{r}) \hat{\mathbf{z}}, t^{v}(\mathbf{r})$ being the total field, at each $v$ th illumination complies with the data equation expressed in terms of the multipole expansion in cylindrical harmonics [30, $\underline{34}, \underline{35}]$,

$$
e^{v}(\mathbf{r})=\sum_{n=1}^{N}\left[H_{0}^{(1)}\left(\frac{2 \pi}{\lambda}\left|\mathbf{r}-\mathbf{r}_{n}\right|\right) a_{n}^{v}\right] \quad \mathbf{r} \in \Omega_{\mathrm{obs}}
$$

where $H_{0}^{(1)}(\cdot)$ is the zeroth-order Hankel function of the first kind, $\Omega_{\mathrm{obs}}$ is the observation domain external to $\Omega$ $\left(\Omega \cap \Omega_{\text {obs }}=\varnothing\right), \lambda$ is the free-space wavelength, and $\mathbf{a}^{v} \triangleq$ $\left\{a_{n}^{v}, n=1, \ldots, N\right\}$ is the vector of the scattering amplitudes given by [30,33-35]:

$$
\mathbf{a}^{v}=[\operatorname{diag}(\gamma) \cdot \Psi+I]^{-1} \cdot \operatorname{diag}(\gamma) \cdot \Phi \cdot \mathbf{f}^{v},
$$

$I$ being the $N$-by- $N$ identity matrix. (In this paper the classical LSF notation from [30] is adopted in which the auxiliary matrices $\Psi$ and $\Phi$ are known quantities not depending on $\gamma$.) Moreover, the entries of the auxiliary matrices $\Psi \triangleq\left\{\psi_{n p}, n=1, \ldots, N ; p=1, \ldots, N\right\}$ and $\Phi \triangleq\left\{\phi_{n q}, n=\right.$ $1, \ldots, N ; q=-Q, \ldots, Q\}$ are

$$
\psi_{n p}=\left\{\begin{array}{cc}
\frac{J_{0}\left(\frac{2 \sqrt{\pi \delta}}{\lambda}\right) H_{0}^{(1)}\left(\frac{2 \pi}{\lambda}\left|\mathbf{r}_{n}-\mathbf{r}_{p}\right|\right)}{H_{0}^{(1)}\left(\frac{2 \sqrt{\pi \delta}}{\lambda}\right)} & n \neq p \\
0 & n=p
\end{array},\right.
$$

$$
\phi_{n q}=\frac{J_{0}\left(\frac{2 \sqrt{\pi \delta}}{\lambda}\right)}{H_{0}^{(1)}\left(\frac{2 \sqrt{\pi \delta}}{\lambda}\right)} J_{-q}\left(\frac{2 \pi}{\lambda}\left|\mathbf{r}_{n}\right|\right) \exp \left[i q \arctan \left(\frac{y_{n}}{x_{n}}\right)\right],
$$

respectively, $J_{q}(\cdot)$ being the $q$ th-order Bessel function of the first kind; $i$ stands for the imaginary unit. Moreover, $\mathbf{f}^{v} \triangleq$ $\left\{f_{q}^{v}=\exp \left(-i q \theta^{v}\right) ; q=-Q, \ldots, Q\right\}$ is the vector whose elements are the coefficients of the expansion in cylindrical harmonics of the $v$ th incident wave $f^{v}(\mathbf{r})$

$$
f^{v}(\mathbf{r}) \triangleq \sum_{q=-Q}^{Q}\left\{J_{q}\left(\frac{2 \pi}{\lambda}|\mathbf{r}|\right) \exp \left[i q \arctan \left(\frac{y}{x}\right)\right] f_{q}^{v}\right\}
$$

$Q$ being the user-defined truncation index [35].

Starting from a set of $V$ scattered vectors, $\mathbf{e}^{v} \triangleq\left[e^{v}\left(\mathbf{r}_{m}^{v}\right), m=1, \ldots, M\right], v=1, \ldots, V$, being $\mathbf{r}_{m}^{v} \in \Omega_{\mathrm{obs}}, m=$ $1, \ldots, M$ the location of the $m$ th measurement point at the $v$ th view, the inversion is then aimed at estimating the sparsest vector $\gamma$ complying with Eqs. (2) and (3). Toward this end, CS strategies seem to be natural candidate tools thanks to their effectiveness in dealing with sparse unknowns [42-45]. However, they cannot be directly applied because of the nonlinearity of the relationships between the data, $\mathbf{e}^{v}$, and the unknowns, $\gamma$, Eqs. (2) and (3). An alternative approach is then considered starting from the following observations.

- Linearity: according to Eq. (2), the amplitude vector $\mathbf{a}^{v}$ linearly depends on $\mathbf{e}^{v}$.

- Sparsity: since $\gamma_{n}=0$ implies $a_{n}^{v}=0$, but not vice versa Eq. (3)., the vector $\mathbf{a}^{v}$ is always sparser than $\gamma:\left\|\mathbf{a}^{v}\right\|_{\ell_{0}} \leq\|\gamma\|_{\ell_{0}}=S$.

- Retrieved information: the support of $\mathbf{a}^{v}$ (i.e., the set of grid cells of $\Omega$ for which $a_{n}^{v} \neq 0$ ) provides a good estimate of the shape of the probed PECs, $s$ since $a_{n}^{v} \neq 0$ implies $\gamma_{n}=1$, Eq. (3).

Accordingly, the scattering amplitude vectors, $\left\{\mathbf{a}^{v} ; v=1, \ldots, V\right\}$, are first retrieved starting from the measured data, $\left\{\mathbf{e}^{v} ; v=1, \ldots, V\right\}$, then a combination and thresholding step is carried out to yield an estimate of the LSF, $\hat{\gamma}$, from $\left\{\mathbf{a}^{v}, v=1, \ldots, V\right\}$. Mathematically, the inversion problem is recast as a two-step one, where the first step follows.

Scattering-amplitudes retrieval problem: given $\mathbf{e}^{v} \in \mathbb{C}^{M}, \quad v=1, \ldots, V$, find the sparsest vector $\mathbf{a}^{v} \in \mathbb{C}^{N}$, $v=1, \ldots, V$, such that $\mathbf{e}^{v}=\mathcal{H}^{v} \mathbf{a}^{v}$, where

$$
\left.\mathcal{H}^{v} \triangleq\left\{\mathcal{H}^{v}\right\rfloor_{m n}=H_{0}^{(1)}\left(\frac{2 \pi}{\lambda}\left|\mathbf{r}_{m}^{v}-\mathbf{r}_{n}\right|\right), m=1, \ldots, M, n=1, \ldots, N\right\}
$$

is the problem kernel, Eq. (2). Once the $V$ scatteringamplitude vectors have been estimated, $\left\{\hat{\mathbf{a}}^{v} ; v=1, \ldots, V\right\}$, the binary LSF is derived, $\hat{\gamma}$, by addressing the following second step.

LSF Retrieval Problem: given $\hat{\mathbf{a}}^{v} \in \mathbb{C}^{N}, v=1, \ldots, V$, determine the estimate $\hat{\gamma}$ by combining the locations of nonzero amplitude coefficients at the different views $\left(\left|a_{n}^{v}\right| \neq 0\right)$. 


\section{LSF-MT-BCS RECONSTRUCTION METHOD}

In principle, the solution of the scattering-amplitudes retrieval problem can be yielded through any CS-based technique $[39,40]$. However, deterministic implementations based on the minimization of functionals comprising $\ell_{1}$-norm terms [50] are prevented or are very complex in our framework because of the computational unfeasibility of verifying the restricted isometry property for the $\mathcal{H}$ matrix [39]. Accordingly, a Bayesian CS [38,41] formulation is adopted hereinafter, since (i) it does not require the restricted isometry property of $\mathcal{H}$ to be $a$ priori verified and (ii) it easily allows the introduction of the a priori information concerned with the physics of the problem at hand [43]. Following the BCS guidelines, the scattering amplitudes are retrieved by solving the maximum a posteriori probability equation

$$
\hat{\mathbf{A}}^{v} \triangleq \arg \left\{\max _{\mathbf{A}^{v}}\left[\mathcal{P}\left(\mathbf{A}^{v} \mid \mathbf{E}^{v}\right)\right]\right\}, \quad v=1, \ldots, V,
$$

where $\mathbf{A}^{v} \triangleq\left[\mathcal{R}\left(\mathbf{a}^{v}\right), \mathcal{I}\left(\mathbf{a}^{v}\right)\right]^{\prime} \in \mathbb{R}^{2 N}$ and $\mathbf{E}^{v} \triangleq\left[\mathcal{R}\left(\mathbf{e}^{v}\right), \mathcal{I}\left(\mathbf{e}^{v}\right)\right]^{\prime} \in$ $\mathbb{R}^{2 M}$ are the real-valued counterparts of the vectors $\mathbf{a}^{v}$ and $\mathbf{e}^{v}$ (prime being the transpose operator), respectively, and $\mathcal{P}\left(\mathbf{A}^{v} \mid \mathbf{E}^{v}\right)$ is the posterior probability distribution that encodes the prior assumptions on $\mathbf{A}^{v}$ [38]. More specifically, the latter is expressed through the Bayes' theorem

$$
\mathcal{P}\left(\mathbf{A}^{v} \mid \mathbf{E}^{v}\right)=\frac{\mathcal{P}\left(\mathbf{E}^{v} \mid \mathbf{A}^{v}\right) \mathcal{P}\left(\mathbf{A}^{v}\right)}{\mathcal{P}\left(\mathbf{E}^{v}\right)}
$$

in terms of the prior $\mathcal{P}\left(\mathbf{A}^{v}\right)$ to account for (a) the a priori knowledge of the sparseness of the scattering amplitudes, $\mathbf{a}^{v}, v=1, \ldots, V$, and (b) the correlation among the locations of the non-null entries, $a_{n}^{v} \neq 0$, of the scattering vectors at different views. Accordingly, the MT-BCS formulation is adopted by introducing a shared prior:

$$
\mathcal{P}\left(\mathbf{A}^{v}\right) \triangleq \int \mathcal{P}\left(\mathbf{A}^{v} \mid \alpha, \sigma\right) \mathcal{P}(\alpha) \mathcal{P}(\sigma) \mathrm{d} \alpha \mathrm{d} \sigma,
$$

where $\alpha \triangleq\left\{\alpha_{n} ; n=1, \ldots, 2 N\right\}$ is the shared prior (i.e., it does not depend on the illumination index $v$ ) hyperparameter vector.

Following the hierarchical hyperparameter procedure discussed in $[38,43,47]$, Eq. (8) is substituted into Eq. (7) to yield the a posteriori model,

$$
\mathcal{P}\left(\mathbf{A}^{v} \mid \mathbf{E}^{v}\right)=\int \mathcal{P}\left(\mathbf{A}^{v} \mid \mathbf{E}^{v}, \alpha\right) \mathcal{P}\left(\alpha \mid \mathbf{E}^{v}\right) \mathrm{d} \alpha,
$$

for Eq. (ㅁ), and the LSF-MT-BCS solution is obtained according to the steps discussed in [38]:

$$
\hat{\mathbf{A}}^{v}=\left[\operatorname{diag}(\hat{\alpha})+\left(\mathcal{K}^{v}\right)^{\prime} \mathcal{K}^{v}\right]^{-1}\left(\mathcal{K}^{v}\right)^{\prime} \mathbf{E}^{v} \quad v=1, \ldots, V,
$$

where the matrix $\mathcal{K}^{v} \in \mathbb{R}^{2 M \times 2 N}$ is given by $\mathcal{K}^{v} \triangleq\left[\mathcal{J}^{v},-\mathcal{Y}^{v}\right.$; $\left.\mathcal{Y}^{v}, \mathcal{J}^{v}\right]$, for which

$\left.\mathcal{J}^{v} \triangleq\{\mathcal{J}\rfloor_{m n}=J_{0}\left(\frac{2 \pi}{\lambda}\left|\mathbf{r}_{m}^{v}-\mathbf{r}_{n}\right|\right), m=1, \ldots, M, n=1, \ldots, N\right\}$

and
$\left.\mathcal{Y}^{v} \triangleq\{\mathcal{Y}\rfloor_{m n}=Y_{0}\left(\frac{2 \pi}{\lambda}\left|\mathbf{r}_{m}^{v}-\mathbf{r}_{n}\right|\right), m=1, \ldots, M, n=1, \ldots, N\right\}$,

while $Y_{0}(\cdot)$ is the zeroth-order Bessel function of the second kind. As for $\hat{\alpha}$, it is computed by means of the relevance vector machine technique [38] aimed at minimizing, with respect to $\alpha$, the function

$$
\Im(\alpha)=-\frac{1}{2} \sum_{v=1}^{V}\left\{\log (|\mathcal{C}|)+\left(2 N+2 \beta_{1}\right) \log \left[\left(\mathbf{E}^{v}\right)^{\prime} \mathcal{C} \mathbf{E}^{v}+2 \beta_{2}\right]\right\},
$$

where $\mathcal{C} \triangleq I+\mathcal{K}^{v} \operatorname{diag}(\alpha)^{-1}\left(\mathcal{K}^{v}\right)^{\prime}$, while $\beta_{1}$ and $\beta_{2}$ are user defined control parameters.

Once $\hat{\mathbf{A}}^{v}$ has been determined through Eq. (10), the scattering amplitudes (scattering-amplitudes retrieval problem) turn out to be

$$
\left.\left.\hat{\mathbf{a}}^{v} \triangleq\left\{\hat{a}_{n}^{v}=\hat{\mathbf{A}}^{v}\right\rfloor_{n}+i \hat{\mathbf{A}}^{v}\right\rfloor_{n+N}, n=1, \ldots, N\right\} \quad v=1, \ldots, V
$$

As far as the LSF retrieval problem is concerned, the information on the support of $\hat{\mathbf{a}}^{v}$ could be used since it is directly linked to the shape of the PEC, Eq. (3). However, such an approach would be extremely sensitive to small inaccuracies in the reconstruction, since even a small value of $\left|\hat{a}_{n}^{v}\right|$ would

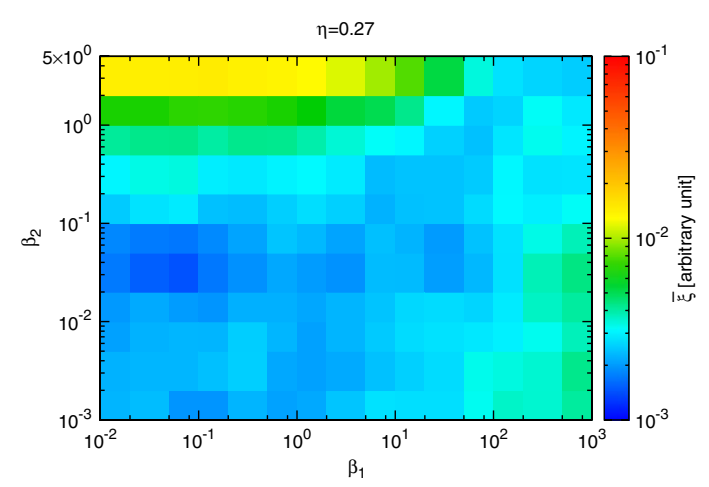

(a)

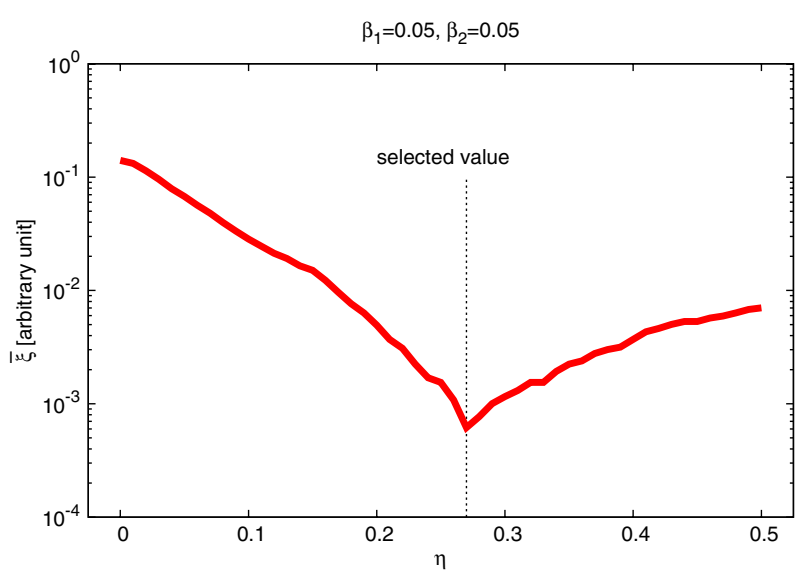

(b)

Fig. 1. LSF-MT-BCS calibration (multiple scatterers, $\ell=\lambda / 6$, $O=S=10$ ). Behavior of the average integral error, $\bar{\xi}$, versus (a) $\beta_{1}$ and $\beta_{2}$ when $\eta=\eta^{\text {opt }}$ and (b) $\eta$ when $\beta_{1}=\beta_{1}^{\text {opt }}$ and $\beta_{2}=\beta_{2}^{\text {opt }}$. 


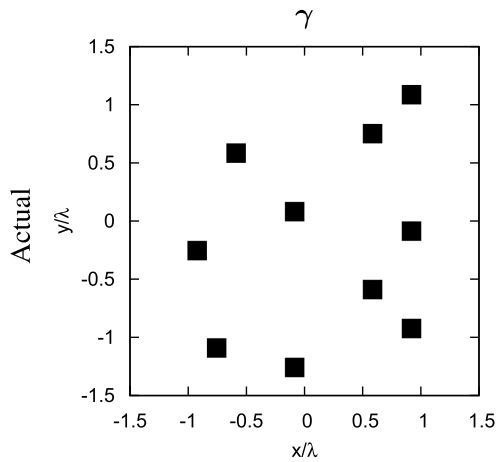

(a)

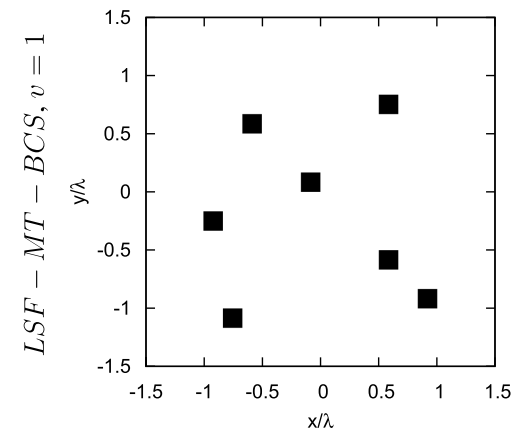

(c)

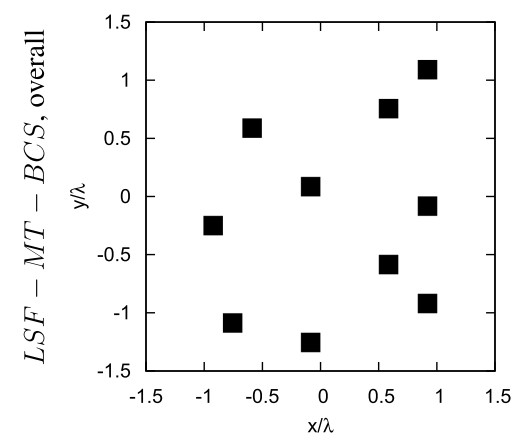

(e)

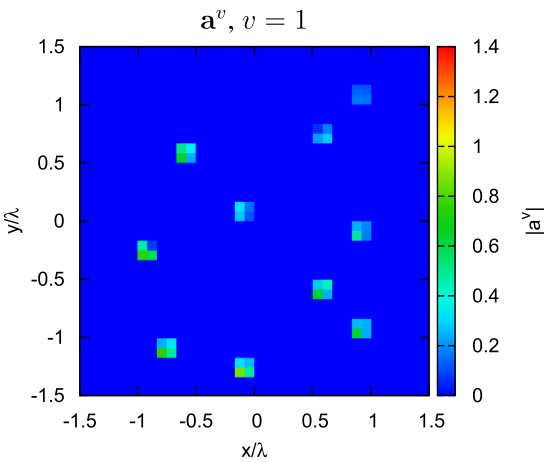

(b)

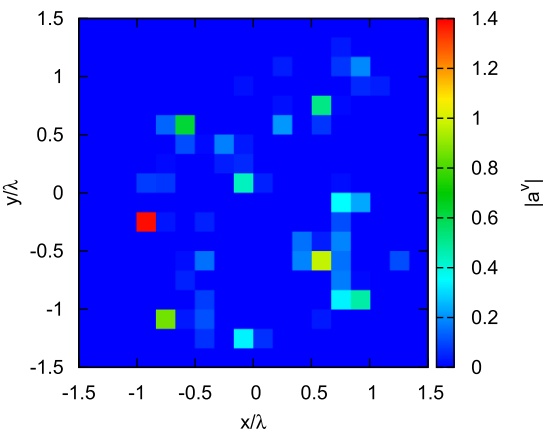

(d)

Fig. 2. Illustrative example (multiple scatterers, $\ell=\lambda / 6, O=S=10$, SNR $=20 \mathrm{~dB}$ ). (a), (c), (e) Binary function $\gamma$ and (b), (d) amplitude coefficients $\mathbf{a}^{v}(v=1)$ of (a), (b) the actual PEC profile and the LSF-MT-BCS (c), (d) single-view $(v=1)$ and (e) multiview $(v=1, \ldots, V)$ reconstructions.

correspond to a presence of a PEC cell. Therefore, the binary LSF coefficients, $\hat{\gamma}_{n}^{v}, n=1, \ldots, N$, associated with the $v$ th view are derived from the distribution of the scattering amplitudes by thresholding

$$
\hat{\gamma}_{n}^{v} \triangleq H\left(\frac{\left|\hat{a}_{n}^{v}\right|}{\max _{n}\left\{\left|\hat{a}_{n}^{v}\right|\right\}}-\eta\right) \quad n=1, \ldots, N
$$

where $H(\cdot)$ is the Heaviside step function and $\eta$ is a userdefined threshold. Finally, the PEC profile (i.e., $\hat{\gamma}_{n}^{v}$, $n=1, \ldots, N)$ is estimated by means of a majority voting rule,

$$
\hat{\gamma}_{n} \triangleq H\left(\frac{\sum_{v=1}^{V} \hat{\gamma}_{n}^{v}}{V}-0.5\right) \quad n=1, \ldots, N
$$

It is worthing pointing out that the procedure proposed here for solving the LSF retrieval problem differs from the combination rule applied in source reconstruction methodologies [37], since (a) a thresholding mechanism is used to minimize artifacts, Eq. (13), (b) a voting rule is exploited, Eq. (14), instead of a linear summation of the retrieved amplitudes, and (c) the binary LSF coefficients $\hat{\gamma}_{n}$ are estimated here instead of the volumetric currents.

\section{NUMERICAL ASSESSMENT}

\section{A. Rationale and Figures of Merit}

A selected set of numerical experiments is discussed hereinafter to provide suitable guidelines for the choice of the LSFMT-BCS control parameters as well as to investigate the method features and potentialities in terms of accuracy, speed, and robustness to noise. Comparisons with the single-task (ST) implementation [42] of the LSF-BCS approach are reported, as well. 


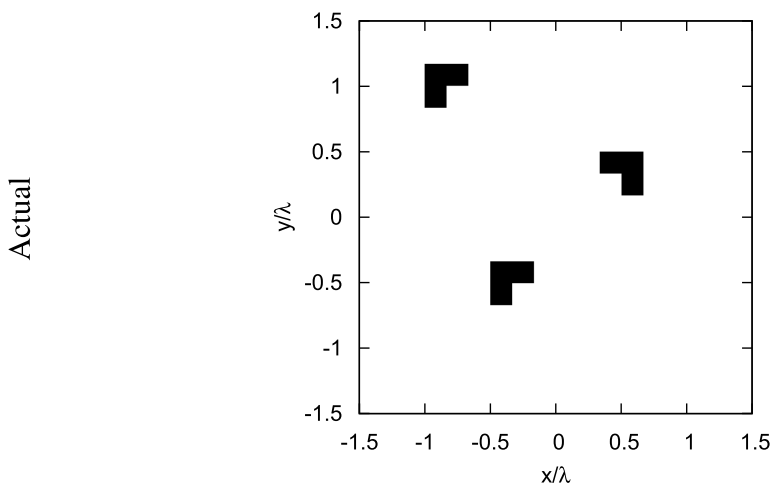

(a)

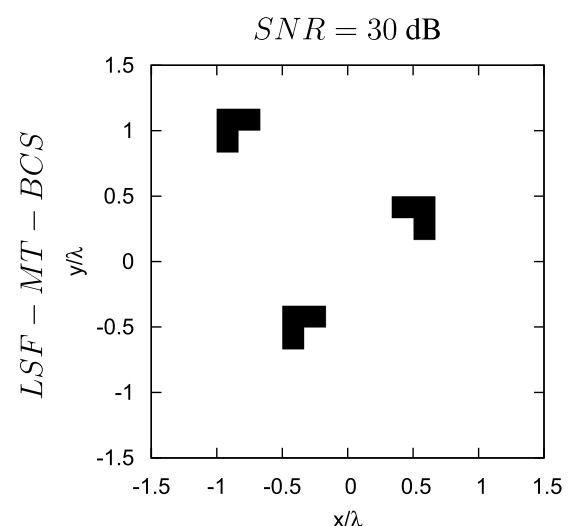

(b)

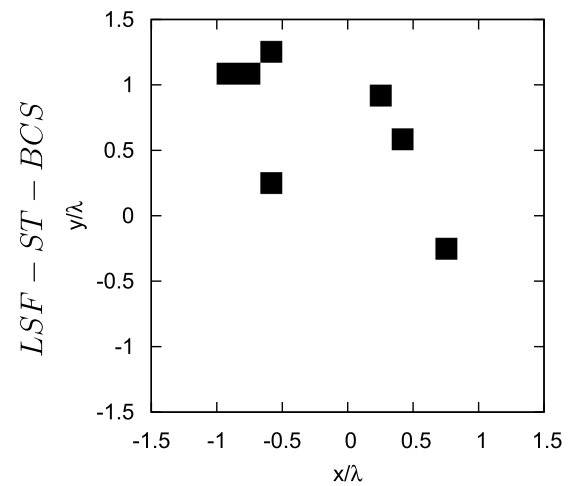

(d)

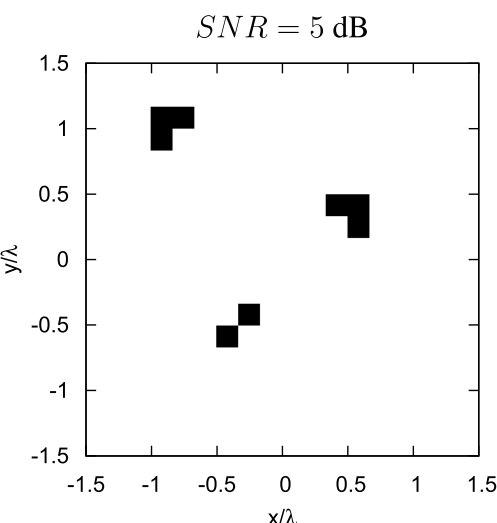

(c)

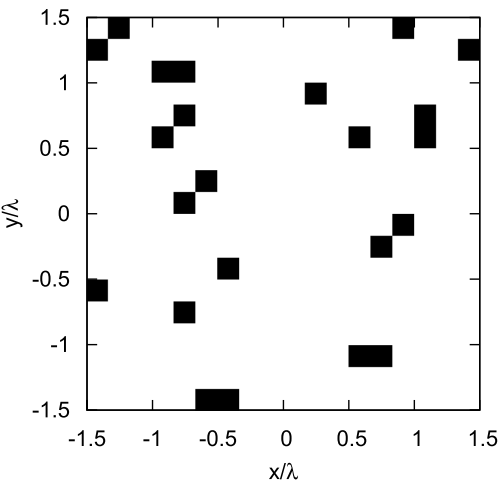

(e)

Fig. 3. Numerical assessment (multiple $L$-shaped scatterers, $O=3, S=9$ ). (a) Actual and (b)-(e) retrieved binary function $\gamma$ with (b), (c) the LSF-MT-BCS and (d), (e) the LSF-ST-BCS when (b), (d) SNR $=30 \mathrm{~dB}$ and (c), (e) SNR $=5 \mathrm{~dB}$.

If not otherwise indicated, the numerical assessment refers to the following benchmark scattering scenario. An $L=3 \lambda_{0^{-}}$ sided square investigation domain $\Omega$ is discretized in $N=324$ cells centered at

$$
\left.\mathbf{r}_{n}=\left[\frac{L}{\sqrt{N}}(n-1)\right\rfloor_{\bmod \sqrt{N}}, \frac{L}{\sqrt{N}}\left\lfloor\frac{(n-1)}{\sqrt{N}}\right\rfloor\right] n=1, \ldots, N
$$

and illuminated by $V=27$ incident waves impinging from the directions

$$
\theta_{v}=\frac{2 \pi(v-1)}{V}, \quad v=1, \ldots, V
$$

The scattering data, $\mathbf{e}^{v}=\left[e^{v}\left(\mathbf{r}_{m}^{v}\right), m=1, \ldots, M\right]$, have been computed (unlike the inversion, a finer grid has been considered for the forward solver to avoid the inverse crime 51.) at $M=27$ locations,

$$
\mathbf{r}_{m}^{v}=\left[\rho \cos \left(\frac{2 \pi(m-1)}{M}\right), \rho \sin \left(\frac{2 \pi(m-1)}{M}\right)\right] \quad m=1, \ldots, M,
$$

of the observations domain, defined over a circle $\rho=3 \lambda_{0}$ in radius, by means of the LSF approach [30], Eqs. (2)-(5), and assuming $Q=30$. 


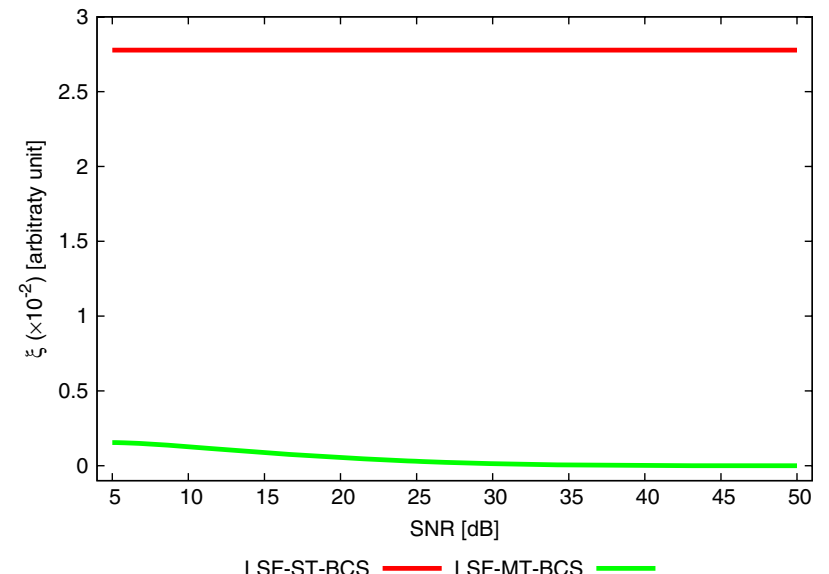

Fig. 4. Numerical assessment (multiple $L$-shaped scatterers, $O=3$, $S=9$ ). Behavior of the integral error $\xi$ versus SNR.

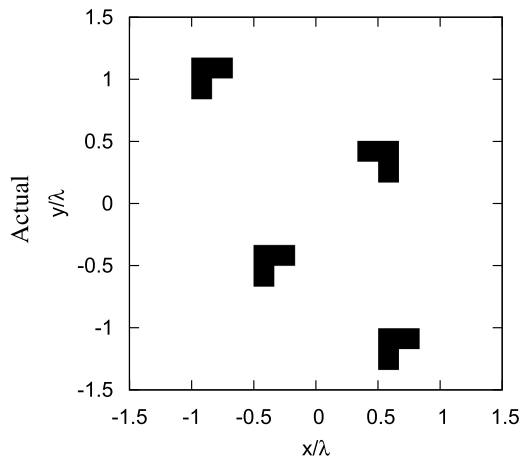

(a)

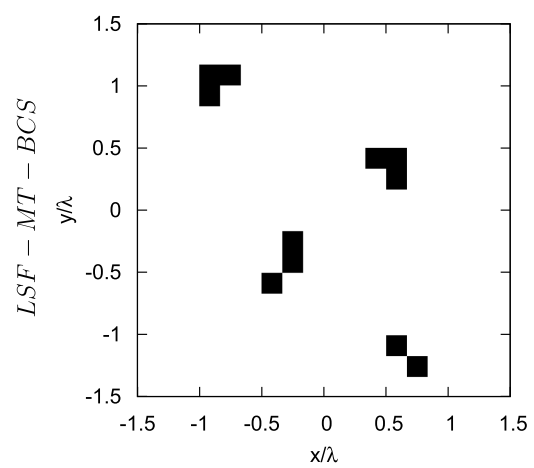

(b)

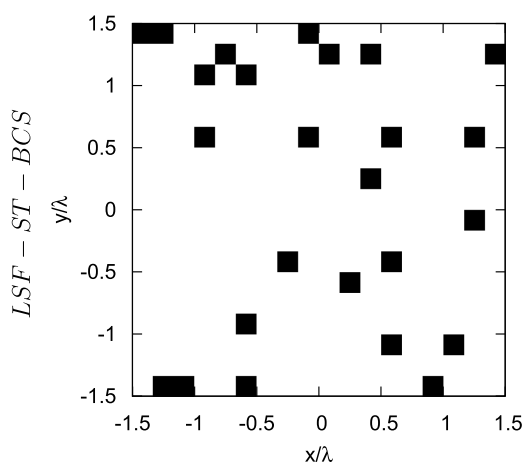

(c)

Fig. 5. Numerical assessment (multiple $L$-shaped scatterers, $O=4$, $S=12$, SNR $=5 \mathrm{~dB}$ ). (a) Actual and retrieved binary function $\gamma$ with (b) the LSF-MT-BCS and (c) the LSF-ST-BCS.

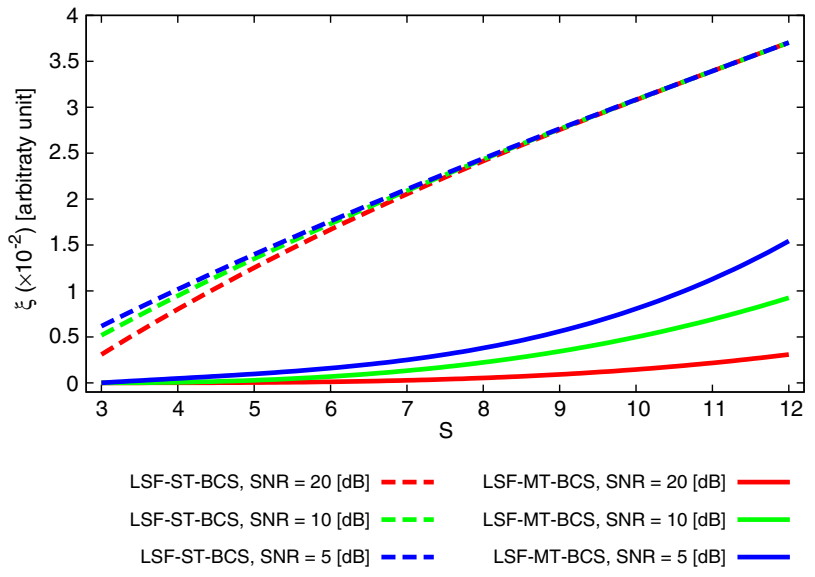

Fig. 6. Numerical assessment (multiple $L$-shaped scatterers, SNR $\in\{5,10,20\} \mathrm{dB}$ ). Behavior of the integral error $\xi$ versus $S$.

In addition to the graphical representation of the retrieved PEC profile, $\hat{\gamma}_{n}, n=1, \ldots, N$, and to provide a qualitative indication on the reconstruction accuracy, the values of the integral error

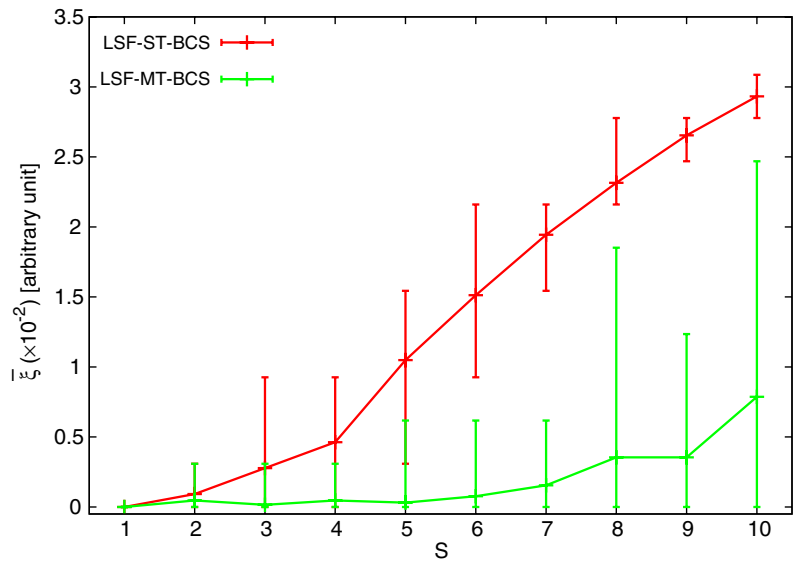

(a)

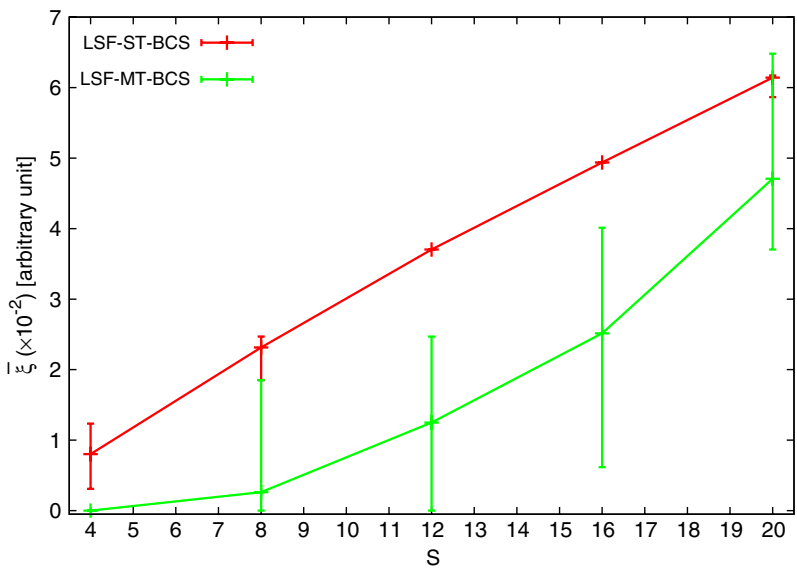

(b)

Fig. 7. Numerical assessment (statistical analysis, $\mathrm{SNR}=10 \mathrm{~dB}$ ). Behavior of the average (points) and maximum and minimum (error bars) values of the integral error $\xi$ versus $S$ in the presence of (a) $\ell=\lambda / 6$ and (b) $\ell=\lambda / 3$ scatterers. 


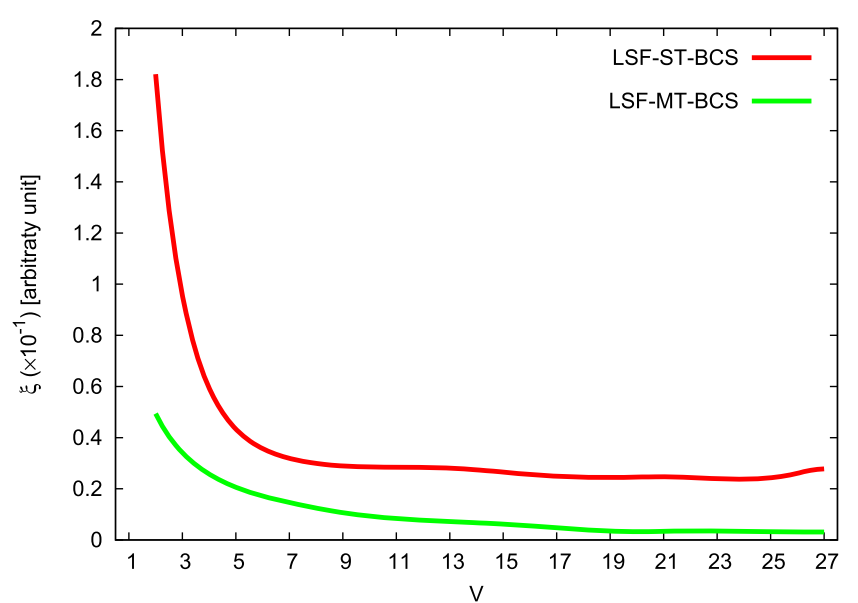

(a)

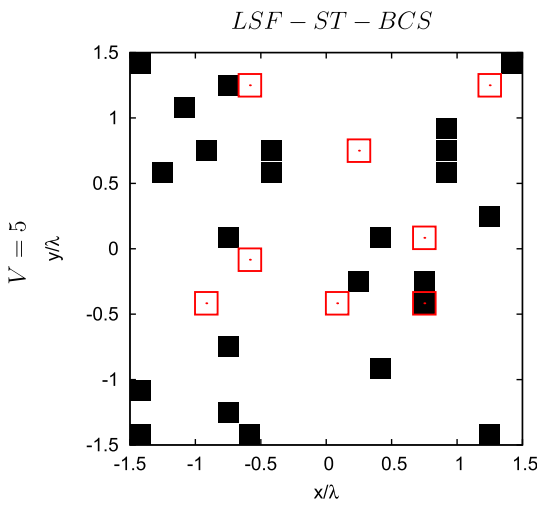

(b)

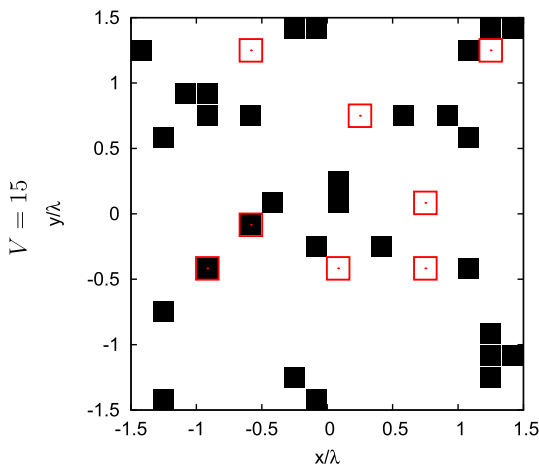

(d)

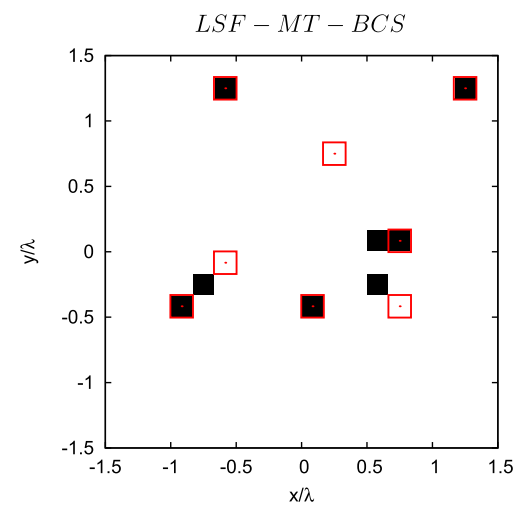

(c)

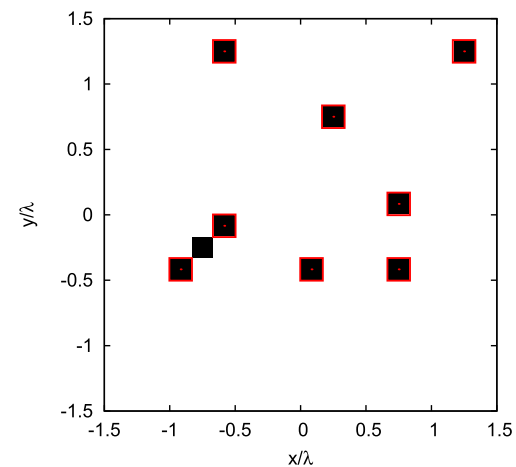

(e)

Fig. 8. Numerical assessment (multiple squares profile, $S=8, \ell=\lambda / 6$, SNR $=10 \mathrm{~dB}$ ). (a) Behavior of the integral error $\xi$ versus $V$ when $M=27$. (b)-(e) Reconstructed distributions when (b), (c) $V=5$ and (d), (e) $V=15$ by applying (b), (d) the LSF-ST-BCS and (c), (e) the LSF-MT-BCS.

$$
\xi \triangleq \frac{1}{N} \frac{\sum_{n=1}^{N}\left|\hat{\gamma}_{n}-\gamma_{n}\right|^{2}}{\sum_{n=1}^{N}\left|\gamma_{n}\right|^{2}}=\frac{\sum_{n=1}^{N}\left|\hat{\gamma}_{n}-\gamma_{n}\right|^{2}}{N \times S}
$$

have been also computed and reported.

\section{B. Control Parameters-Selection Guidelines}

A calibration of the control parameters of the proposed inversion is mandatory to provide suitable guidelines to the interested reader. As a matter of fact, the setup of the control parameters $\beta_{1}$ and $\beta_{2}$ in Eq. (11) is known to be a fundamental step to guarantee the MT-BCS effectiveness and efficiency, since no physical hints are available for their optimal choice [43]. Moreover, the value of the threshold $\eta$, Eq. (13), must guarantee a good filtering-accuracy trade-off whatever the PEC inversion at hand.

Toward this purpose, a set of 100 different scenarios comprising $O=S=10$ randomly displaced square cylinders $\lambda / 6$-sided [e.g., Fig. 2(a)] has been generated, and the LSF-MTBCS inversion has been performed by varying the control parameters within the ranges $\beta_{1} \in\left[10^{-2}, 10^{3}\right], \beta_{1} \in\left[10^{-3}, 5\right]$, and $\eta \in[0,0.5]$. To provide statistical relevance, a set of different signal-to-noise ratios (SNRs) in the range $\mathrm{SNR} \in$ [5 dB, $30 \mathrm{~dB}]$ has been considered, as well. 


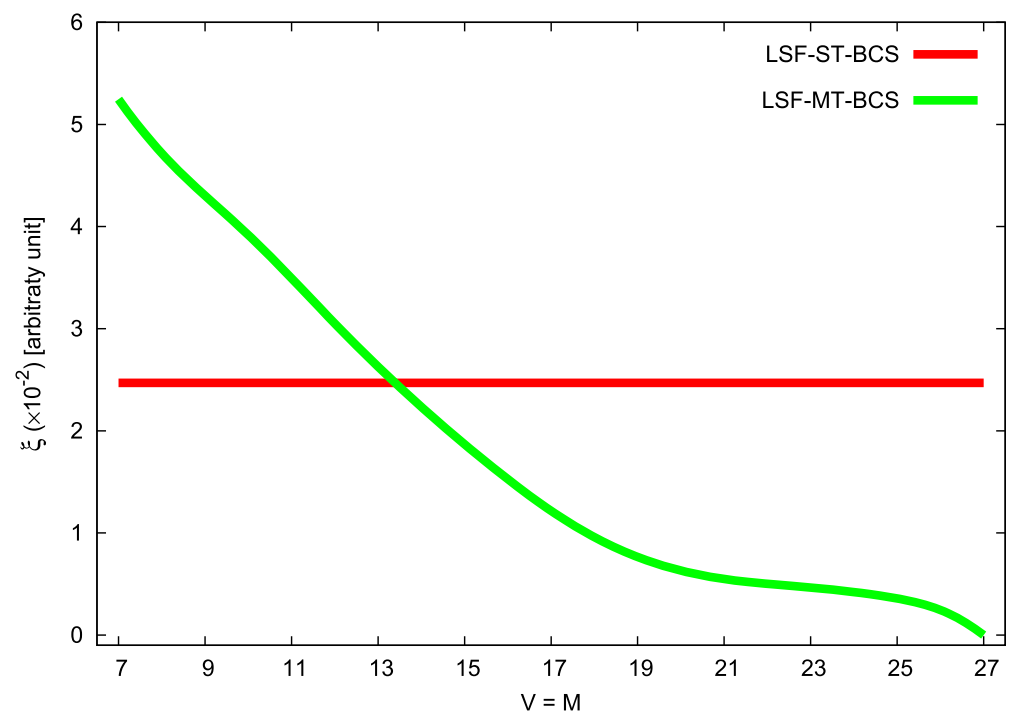

(a)

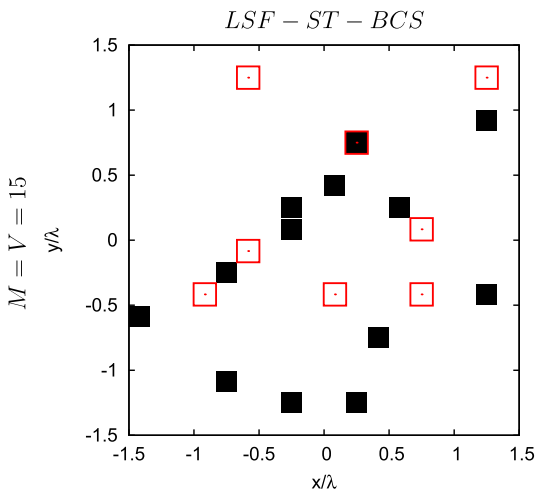

(b)

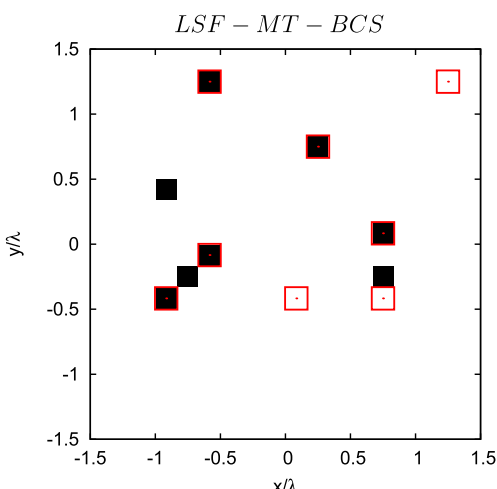

(c)

Fig. 9. Numerical assessment (multiple squares profile, $S=8, \ell=\lambda / 6$, SNR $=10 \mathrm{~dB}$ ). (a) Behavior of the integral error $\xi$ versus the total number of measurement points when $V=M$. Reconstructed distributions when $M=V=15$ by applying (b) the LSF-ST-BCS and (c) the LSF-MT-BCS.

The behavior of the averaged integral error, $\bar{\xi}$, as a function of the hyperparameter control variables [Fig. 1(a)] shows that the accuracy decreases in the regions $\beta_{2} \geq 2.0$ and $\beta_{1} \leq 10$ [e.g., $\bar{\xi} \approx 2.13 \times 10^{-2}$ when $\beta_{1}=0.1, \beta_{2}=5.0$, Fig. $\left.1(\mathrm{a})\right]$ or $\beta_{1} \geq 500$ and $\beta_{2} \leq 10^{-1}$ [e.g., $\bar{\xi} \approx 5.8 \times 10^{-3}$ when $\beta_{1}=500$, $\beta_{2}=2.0 \times 10^{-2}$, Fig. 1(a)], while the minimum $\bar{\xi}$ [i.e., $\bar{\xi}^{\text {opt }} \approx 9.2 \times 10^{-4}$, Fig. 1 (a)] occurs in correspondence with the values $\beta_{1}^{\text {opt }} \approx 5 \times 10^{-2}$ and $\beta_{2}^{\text {opt }} \approx 5 \times 10^{-2}$. These latter values will be assumed hereinafter as the optimal trade-off calibration setting.

As for the behavior of $\bar{\xi}$ versus $\eta$ when assuming $\beta_{1}=\beta_{1}^{\text {opt }}$ and $\beta_{2}=\beta_{2}^{\text {opt }}$, the plot in Fig. 1(b) indicates that a small threshold value yields a poor reconstruction accuracy (e.g., $\bar{\xi}\rfloor_{\eta=2.0 \times 10^{-2}} \approx 1.13 \times 10^{-1} \gg \bar{\xi}^{\text {opt }}$ ) since $\hat{a}_{n}^{v} \approx 0 \rightarrow \hat{\gamma}_{n}=1$. On the other hand, larger values of $\eta$ also tend to filter-out relevant information (e.g., $\bar{\xi}\rfloor_{\eta=4.7 \times 10^{-1}} \approx 5.91 \times 10^{-3} \gg \bar{\xi}^{\text {opt }}$ ). Therefore, the intermediate value $\eta^{\text {opt }} \approx 0.27$, corresponding to the abscissa of the minimum in Fig. 1(b), has been chosen, and it will be used in the following.

For completeness, the plot of the scattering amplitudes $\hat{\mathbf{a}}^{v}$ when $v=1$ and $\mathrm{SNR}=20 \mathrm{~dB}$ for a representative scenario within the set of the calibration simulations [Fig. 2(d)] indicate that, although the LSF amplitudes are only roughly retrieved [Fig. 2(b) versus Fig. 2(d)], their support is very close to the actual object area. Thus, the thresholded version obtained through Eq. (13) exhibits only minor inaccuracies [Fig. 2(a) versus Fig. 2(c)], and the combination of the estimation outcomes from different views, Eq. (14), gives a perfect reconstruction of the scenario under test [Fig. 2(e)].

\section{Numerical Assessment and Comparisons}

The experiments in this section are also aimed at assessing the features and the performances of the proposed technique through comparisons with the ST-BCS [42] implementation. The first test case deals with $O=3 \bar{L}$-shaped cylinders $[S=9$, Fig. $3(\mathrm{a})]$ under various noisy conditions $\mathrm{SNR} \in$ $[5 \mathrm{~dB}, 50 \mathrm{~dB}]$. With reference to the case $\mathrm{SNR}=30 \mathrm{~dB}$, the plot of the LSF-MT-BCS reconstruction in Fig. 3(b) shows that the method perfectly retrieves the scatterer locations and profiles, still keeping the efficiency proved in Fig. 2 despite the size increase of the scatterers. Moreover, only minor errors arise in heavier conditions: $\xi\rfloor_{\mathrm{SNR}=5 \mathrm{~dB}} \approx 1.5 \times 10^{-3}$ [Fig. 3(c)], while the ST version becomes worse whatever the $\mathrm{SNR}$ [e.g., $\xi\rfloor_{\mathrm{SNR}=30 \mathrm{~dB}} \approx 2.77 \times 10^{-2}$, Fig. $3(\mathrm{~d})$ ]. Such an 


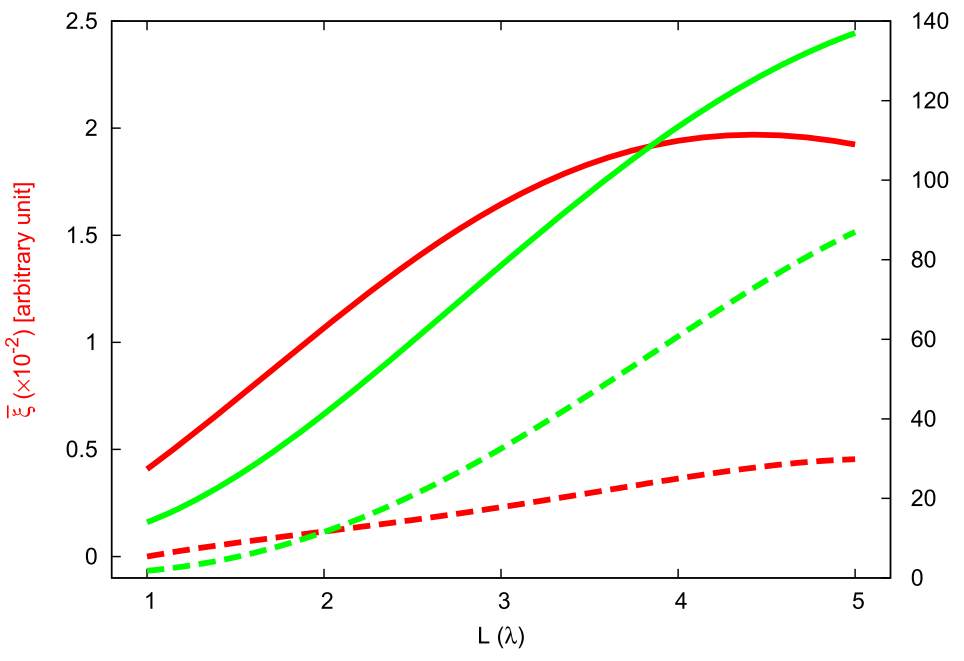

LSF-ST-BCS LSF-ST-BCS LSF-MT-BCS $=$ L $=$ LSF-MT-BCS $=-\mathbf{-}$

(a)

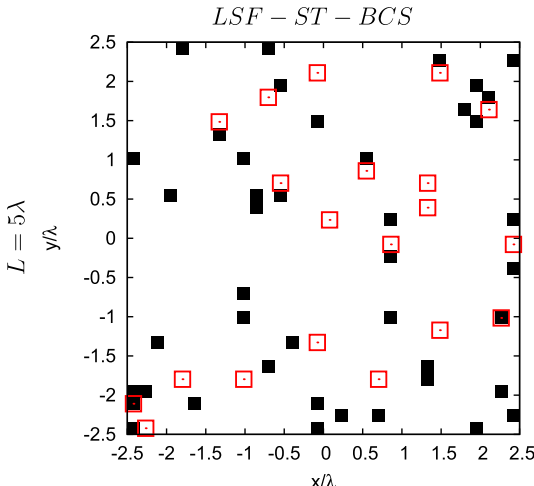

(b)

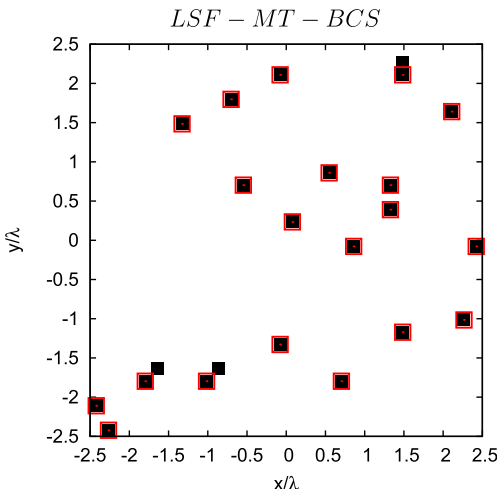

(c)

Fig. 10. Numerical assessment (multiple squares profiles, $\ell=\lambda / 6, S \approx N / 50$, SNR $=10 \mathrm{~dB}$ ). (a) Behavior of the integral error $\xi$ and the CPU time versus $L \in[\lambda, 5 \lambda]$. (b), (c) Reconstructed distributions when $L=5 \lambda$ by applying (b) the LSF-ST-BCS and (c) the LSF-MT-BCS.

outcome is actually expected, since the LSF-ST-BCS encodes a sparsity prior that does not take into account the correlation among the locations of the $a_{n}^{v} \neq 0$ amplitudes at different views [르]. Therefore, the estimated vectors $\hat{\mathbf{a}}^{v}(v=1, \ldots, V)$ turn out to be mutually independent, and their multiview combination generally does not improve the reconstruction with respect to the single-view case. Previous outcomes are also confirmed by the behavior of $\xi$ versus the SNR for the two approaches (Fig. 4). The reconstruction error of the LSF-MT-BCS is always the lowest, and it becomes negligible $(\xi \rightarrow 0)$ when SNR $\geq 30 \mathrm{~dB}$.

Similar conclusions hold true also in the presence of a larger number of scatterers $[O=4-$ Fig. 5(a)]. As an example and despite the high noise level [SNR $=5 \mathrm{~dB}$, Fig. 5(b)], the MT technique still identifies the presence and the positions of the scatterers, unlike the LSF-ST-BCS technique, whose prediction is totally unsatisfactory and has several artifacts [Fig. 5(c)]. This further highlights the importance of exploiting within the inversion process the correlation among different views, rather than doing it only a posteriori.

On the other hand, it has also to be observed that a less sparse distribution of the scatterers affects the method's performance whatever the BCS implementation, as shown by the plots in Fig. $\underline{6}$, and is concerned with the integral error for different scenarios including from $O=1(S=3)$ up to $O=4(S=12) L$-shaped scatterers. Although the MT-based inversion always overcomes the ST and a smoother performance degradation arises, there is a reduction of the fidelity of the LSF-MT-BCS reconstruction when $S$ increases, as well.

To fully assess the sensitivity of the LSF-BCS-based inversion on the sparsity of the PECs, the successive test case deals with $O \in[1,10] \lambda / 6$-sided objects randomly located within $\Omega$. For a choice of 30 trial scatterer configurations for each $O$ value, the statistics (i.e., average, minimum, and maximum values) of the integral error are reported in Fig. 7(a) $(\mathrm{SNR}=10 \mathrm{~dB})$. As it can be observed, the inversion accuracy of the LSF-MT-BCS is reduced on average with $S$ (e.g., $\bar{\xi}\rfloor_{S=6} \approx$ $7.71 \times 10^{-4}$ versus $\left.\bar{\xi}\right\rfloor_{S=10} \approx 7.87 \times 10^{-3}$ ), while the error range is enlarged when less sparse profiles are at hand $\left.\left[\left(\xi_{\max }-\xi_{\min }\right)\right\rfloor_{S=6} \approx 6.2 \times 10^{-3} \operatorname{versus}\left(\xi_{\max }-\xi_{\min }\right)\right\rfloor_{S=10} \approx 2.4 \times$ $\left.10^{-2}\right]$. This indicates that the reconstruction reliability improves for lower $S$ values whatever the target positions as a consequence of the hypotheses on the prior specification, Eq. (8). To confirm such a deduction, the retrieval of larger randomly displaced PECs has been then addressed $[\ell=\lambda / 3$-sided objects-Fig. 7(b)]. As expected, a similar trend of the average, minimum, and maximum errors can 
be noticed [e.g., $\bar{\xi}]_{S=8}^{\ell=\lambda / 6} \approx 3.53 \times 10^{-3}$, Fig. $\underline{7(\text { a) }}$, versus $\bar{\xi}\rfloor_{S=8}^{\ell=\lambda / 3} \approx 2.63 \times 10^{-3}$, Fig. $\underline{7(\mathrm{~b})}$ ]. Throughout the analysis, the figures of merit of the ST approach are also reported to further assess the improvements of the MT-BCS over the ST-BCS inversion [Figs. 7(a) and 7(b)].

The next set of experiments is devoted to analyzing the dependence of the methods' performances on the number of scattering data when different (i.e., smaller) from the optimal bound discussed in [51] and previously adopted. Indeed, CS-based methodologies are known to effectively deal with few data [38-41], as well. Toward this end, the inversion of $O=S=8 \bar{\lambda} / 6$-sided objects [red squares, Fig. 8(b)], randomly distributed within $\Omega$, has been carried out by successively probing the scenario with a different number of views from $V=1$ up to $V=27$, Eq. (16), but always collecting the scattered field samples at the $\bar{M}=27$ measurement points located as in Eq. (17). The LSF-MT-BCS estimated profiles in Fig. 8 $(\mathrm{SNR}=10 \mathrm{~dB})$ turn out to be similar to the actual one starting from only $V=5$ illuminations $\left[\xi \approx 1.85 \times 10^{-2}\right.$, Fig. $\underline{8(\mathrm{c})}$ ] , that is, considering $80 \%$ fewer data than those suggested by the criterion [51] (i.e., $V_{\text {Nyquist }}=27$ ). Moreover, just $V=15$ views $\left(V / V_{\text {Nyquist }} \approx 55 \%\right)$ are required in order to yield a high-quality reconstruction $\left[\xi \approx 3.08 \times 10^{-3}\right.$, Fig. $\left.8(\mathrm{e})\right]$. These results point to LSF-MT-BCS as an enabling tool for nontrivial savings of acquisition time and complexity with respect to traditional inversion strategies. In contrast, ST reconstructions are generally unsatisfactory [Figs. $\underline{8(b)}$ and $\underline{8(d)}$ ], as is confirmed by the corresponding integral errors [Fig. 8(a)], as well. Let us now analyze the method performance versus the number of both measurement points and illuminations (Fig. 9). By assuming $M=V \in[1,27]$ [SNR $=10 \mathrm{~dB}$, Fig. $\underline{9(\mathrm{a})}$ ], it turns out that an $\xi$ value of the same order of that in Fig. 8(c) [i.e., $\xi \approx 1.85 \times 10^{-2}$, Fig. 9(c)] is obtained when processing at least $M \times V=225$ samples (i.e., $M=V=15$ ). Such an amount of scattering data is greater than that used for Fig. 8(c) (i.e., $M \times V=135)$. This suggests that a contemporary reduction of measurement points and illuminations, when scattering data are uniformly collected within $\Omega_{\text {obs }}$, can be less effective than only the reduction of $V$.

The final set of numerical experiments is aimed at assessing the dependence of the inversion accuracy on the size of the investigation domain $\Omega$. Accordingly, $L$ has been varied within the range $L \in[\lambda, 5 \lambda]$ by setting $N, M$, and $V$ according to the criterion in [51]. Moreover, $S \approx N / 50 \lambda / 6$ sided scatterers have been randomly displaced to keep the same degree of sparsity whatever the $\Omega$ extension. The plot of $\bar{\xi}$ in Fig. $10(\mathrm{a})(\mathrm{SNR}=10 \mathrm{~dB})$ shows that the LSFMT-BCS accuracy slightly deteriorates when $L$ is larger (e.g., $\xi\rfloor_{L=2 \lambda}=1.47 \times 10^{-3}$ versus $\left.\xi\right\rfloor_{L=5 \lambda}=4.54 \times 10^{-3}$ ), but it still remains acceptable when the largest $L$ is reached $[L=5 \lambda$, Fig. $10(\mathrm{c})]$. In contrast, the ST-based approach is totally unreliable in the same case [i.e., $\xi]_{L=5 \lambda}^{\mathrm{ST}}=1.92 \times 10^{-2}$, Fig. 10(b)].

As for the computational issues, the behavior of the inversion time, $\Delta t$, on a single-core laptop $\mathrm{PC}$ running at $2.66 \mathrm{GHz}$ indicates that the MT procedure is also more efficient whatever the $L$ value [e.g., $\Delta t]_{L=4 \lambda}^{\mathrm{ST}} \approx 1.21 \times 10^{2} \mathrm{~s}$ versus $\Delta t\rfloor_{L=4 \lambda}^{\mathrm{MT}} \approx 6.60 \times 10^{1}$, Fig. $\underline{10(\mathrm{a})}$ ]. Moreover, LSF-MT-BCS also needs less than $90 \mathrm{~s}$ for inversion completion [i.e., $\Delta t\rfloor_{L=5 \lambda}^{\mathrm{MT}} \approx 8.71 \times 10^{1} \mathrm{~s}$, Fig. $\left.10(\mathrm{a})\right]$ when dealing with highdimensional spaces (e.g., $N \overline{\rfloor_{L=5 \lambda}}=1024$ ).

\section{CONCLUSIONS}

In this paper, the localization of sparse metallic targets has been addressed by means of a new technique that models thin targets through the LSF approach and solves the inversion problem in a BCS sense, also combining the information from different illuminations within the reconstruction process. More in detail, sparse PECs inversion has been recast as a two-step procedure, where the first step is aimed at retrieving the so-called LSF scattering amplitudes through a MT-BCS technique, Eq. (12), while the second is concerned with a thresholding and voting operation, Eqs. (13) and (14). A set of numerical experiments has been discussed to assess the LSF-MT-BCS technique as well as to analyze its potential and limitations.

As for the main methodological novelties, the paper proposes: (a) an inversion technique for sparse PEC targets based on a two-step LSF-based approach that includes a probabilistic sparsity regularizer and (b) a suitable and innovative (unlike its previous dielectric-oriented applications $[42,44,45])$ exploitation of the BCS to image metallic profiles.

The numerical analysis has pointed out the following issues. (i) It is possible to configure the LSF-MT-BCS control parameters so that reliable inversions can be carried out for a large set of scattering scenarios, noise levels, and measurement setups. (ii) The method yields satisfactory reconstructions when dealing with multiple targets occupying several pixels and low SNRs, as well, although the resulting accuracy depends on the scatterer sparsity. Indeed, owing to the sparsity assumption and the LSF formulation, the retrieval of objects consisting of a very large number of cells is prevented. (iii) Thanks to the BCS framework, the approach also provides satisfactory performance when the number of illuminations or measurements is significantly below the optimal one [51]. (iv) The LSF-MT-BCS technique is computationally more efficient and much more effective than the ST implementation.

Future developments, out of the scope of this paper, will be devoted to investigate the optimal field-probe arrangement (i.e., $\mathbf{r}_{m}^{v}, m=1, \ldots, M, v=1, \ldots, V$ ) to further simplify the LSF-MT-BCS imaging setup while guaranteeing faithful and reliable reconstruction. Extension to $3 \mathrm{D}$ problems is also currently under investigation.

\section{ACKNOWLEDGMENTS}

The authors thank Dr. S. Ji, Dr. Y. Xue, and Prof. L. Carin for sharing the BCS code online [52].

\section{REFERENCES}

1. J. Shen, X. Chen, Y. Zhong, and L. Ran, "Inverse scattering problem in presence of a conducting cylinder," Opt. Express 19, 10698-10706 (2011).

2. K. Agarwal, X. Chen, and Y. Zhong, "A multipole-expansion based linear sampling method for solving inverse scattering problems," Opt. Express 18, 6366-6381 (2010).

3. A. Liseno and R. Pierri, "Imaging perfectly conducting objects as support of induced currents: Kirchhoff approximation and frequency diversity,” J. Opt. Soc. Am. A 19, 1308-1318 (2002).

4. A. Liseno, R. Pierri, and F. Soldovieri, "Shape identification by physical optics: the two-dimensional TE case,” J. Opt. Soc. Am. A 20, 1827-1830 (2003).

5. T. Dorney, W. Symes, R. Baraniuk, and D. Mittleman, "Terahertz multistatic reflection imaging," J. Opt. Soc. Am. A 19, 1432-1442 (2002). 
6. Y.-C. Chen, Y.-F. Chen, C.-C. Chiu, and C.-Y. Chang, "Image reconstruction of a buried perfectly conducting cylinder illuminated by transverse electric waves," Int. J. Imaging Syst. Technol. 15, 261-265 (2005).

7. W. Chien and C.-C. Chiu, "Using NU-SSGA to reduce the searching time in inverse problem of a buried metallic object," IEEE Trans. Antennas Propag. 53, 3128-3134 (2005).

8. A. Schatzberg and A. Devaney, "Rough surface inverse scattering within the Rytov approximation,” J. Opt. Soc. Am. A 10, 942-950 (1993).

9. R. Pierri, R. Solimene, A. Liseno, and J. Romano, "Linear distribution imaging of thin metallic cylinders under mutual scattering," IEEE Trans. Antennas Propag. 53, 3019-3029 (2005).

10. A. Brancaccio, G. Leone, and R. Solimene, "Fault detection in metallic grid scattering," J. Opt. Soc. Am. A 28, 2588-2599 (2011).

11. E. P. Ribeiro, A. C. Bruno, P. C. Ribeiro, J. Szczupak, and O. G. Symko, "Image of a two-dimensional magnetic moment distribution: application to detect small metallic objects in the human body," in 1992 14th Annual International Conference of the IEEE Engineering in Medicine and Biology Society (IEEE, 1992), Vol. 5, 2178-2179.

12. D. Asteli and B. Ottersten, "The effect of local scattering on direction of arrival estimation with MUSIC," IEEE Trans. Signal Process. 47, 3220-3234 (1999).

13. E. Cekli and H. A. Cirpan, "Unconditional maximum likelihood approach for localization of near-field sources: algorithm and performance analysis,” J. Inst. Electron. Commun. Eng. Jpn. 57, 9-15 (2003).

14. K. J. Langenberg, K. Mayer, A. Zimmer, and C. Kohl, "Nondestructive evaluation of embedded structures in concrete: modeling and tomographic imaging," in Proceedings of the URSI International Symposium on Electromagnetic Theory (URSI EMTS, 2004), pp. 1203-1205.

15. R. E. Kleinman and P. M. van den Berg, "Two-dimensional location and shape reconstruction,” Radio Sci. 29, 1157-1169 (1994).

16. F. K. Gruber, E. A. Marengo, and A. J. Devaney, "Time-reversal imaging with multiple signal classification considering multiple scattering between the targets," J. Acoust. Soc. Am. 115, 3042-3047 (2004)

17. T. Miwa and I. Arai, "Super-resolution imaging for point reflectors near transmitting and receiving array," IEEE Trans. Antennas Propag. 52, 220-229 (2004)

18. G. Micolau, M. Saillard, and P. Borderies, "DORT method as applied to ultrawideband signals for detection of buried objects," IEEE Trans. Geosci. Remote Sens. 41, 1813-1820 (2003).

19. P. Rocca, M. Benedetti, M. Donelli, D. Franceschini, and A Massa, "Evolutionary optimization as applied to inverse scattering problems," Inverse Problems 25, 1-41 (2009).

20. P. Rocca, G. Oliveri, and A. Massa, "Differential evolution as applied to electromagnetics," IEEE Antennas Propag. Mag. 53, 38-49 (2011).

21. C.-C. Chiu, C.-H. Sun, C.-L. Li, and C.-H. Huang, "Comparative study of some population-based optimization algorithms on inverse scattering of a two-dimensional perfectly conducting cylinder in dielectric slab medium," IEEE Trans. Geosci. Remote Sens. 51, 2302-2315 (2013).

22. Y. Zhou, J. Li, and H. Ling, "Shape inversion of metallic cavities using hybrid genetic algorithm combined with tabu list," Electron. Lett. 39, 280-281 (2003).

23. A. Qing, "Electromagnetic inverse scattering of multiple twodimensional perfectly conducting objects by the differential evolution strategy," IEEE Trans. Antennas Propag. 51, 1251-1262 (2003).

24. I. T. Rekanos, "Shape reconstruction of a perfectly conducting scatterer using differential evolution and particle swarm optimization," IEEE Trans. Geosci. Remote Sens. 46, 19671974 (2008).

25. R. Pierri, A. Liseno, R. Solimene, and F. Soldovieri, "Beyond physical optics SVD shape reconstruction of metallic cylinders," IEEE Trans. Antennas Propag. 54, 655-665 (2006).
26. C. Ramananjaona, M. Lambert, and D. Lesselier, "Shape inversion from TM and TE real data by controlled evolution of level sets," Inverse Probl. 17, 1585-1595 (2001).

27. R. Solimene, A. Brancaccio, J. Romano, and R. Pierri, "Localizing thin metallic cylinders by a 2.5-D linear distributional approach: experimental results," IEEE Trans. Antennas Propag. 56, 2630-2637 (2008).

28. D. Colton and P. Monk, "A novel method for solving the inverse scattering problem for time-harmonic acoustic waves in the resonance region II," SIAM J. Appl. Math. 46, 506-523 (1986).

29. K. A. Michalski, "Electromagnetic imaging of circularcylindrical conductors and tunnels using a differential evolution algorithm," Microw. Opt. Technol. Lett. 27, 330-334 (2000).

30. W. C. Chew and G. P. Otto, "Microwave imaging of multiple conducting cylinders using local shape functions," IEEE Microw. Guided Wave Lett. 2, 284-286 (1992).

31. A. Qing and C. K. Lee, "Microwave imaging of parallel perfectly conducting cylinders using real-coded genetic algorithm coupled with Newton-Kantorovich method" Prog. Electromagn. Res. 28, 275-294 (2000).

32. A. Qing, C. K. Lee, and L. Jen, "Electromagnetic inverse scattering of two-dimensional perfectly conducting objects by realcoded genetic algorithm," IEEE Trans. Geosci. Remote Sens. 39, 665-676 (2001)

33. W. C. Chew, L. Gurel, Y.-M. Wang, G. Otto, R. L. Wagner, and Q. H. Liu, "A generalized recursive algorithm for wave-scattering solutions in two dimensions," IEEE Trans. Microwave Theory Tech. 40, 716-723 (1992).

34. G. P. Otto and W. C. Chew, "Microwave inverse scatteringlocal shape function imaging for improved resolution of strong scatterers," IEEE Trans. Microwave Theory Tech. 42, 137-141 (1994)

35. T. Takenaka, Z. Q. Meng, T. Tanaka, and W. C. Chew, "Local shape function combined with genetic algorithm applied to inverse scattering for strips," Microw. Opt. Technol. Lett. 16, 337-341 (1997).

36. I. T. Rekanos and T. D. Tsiboukis, "An inverse scattering technique for microwave imaging of binary objects," IEEE Trans. Microw. Theory Tech. 50, 1439-1441 (2002).

37. Y. Alvarez-Lopez, A. Dominguez-Casas, C. Garcia-Gonzalez, and F. Las-Heras, "Geometry reconstruction of metallic bodies using the sources reconstruction method," IEEE Antennas Wireless Propag. Lett. 9, 1197-1200 (2010).

38. S. Ji, D. Dunson, and L. Carin, "Multitask compressive sensing," IEEE Trans. Signal Process. 57(4), 92-106 (2009)

39. R. G. Baraniuk, "Compressive sampling," IEEE Signal Process. Mag. 24, 118-121 (2007).

40. E. J. Candes and M. B. Wakin, "An introduction to compressive sampling," IEEE Signal Process. Mag. 25, 21-30 (2008).

41. S. Ji, Y. Xue, and L. Carin, "Bayesian compressive sensing," IEEE Trans. Signal Process. 56, 2346-2356 (2008).

42. G. Oliveri, P. Rocca, and A. Massa, "A Bayesian compressive sampling-based inversion for imaging sparse scatterers," IEEE Trans. Geosci. Remote Sens. 49, 3993-4006 (2011).

43. L. Poli, G. Oliveri, P. Rocca, and A. Massa, "Bayesian compressive sensing approaches for the reconstruction of twodimensional sparse scatterers under TE illuminations," IEEE Trans. Geosci. Remote Sens. 51, 2920-2936 (2013).

44. G. Oliveri, L. Poli, P. Rocca, and A. Massa, "Bayesian compressive optical imaging within the Rytov approximation," Opt. Lett 37, 1760-1762 (2012).

45. L. Poli, G. Oliveri, and A. Massa, "Microwave imaging within the first-order Born approximation by means of the contrast-field Bayesian compressive sensing," IEEE Trans. Antennas Propag. 60, 2865-2879 (2012).

46. G. Oliveri and A. Massa, "Bayesian compressive sampling for pattern synthesis with maximally sparse non-uniform linear arrays," IEEE Trans. Antennas Propag. 59, 467-481 (2011).

47. G. Oliveri, M. Carlin, and A. Massa, "Complex-weight sparse linear array synthesis by Bayesian compressive sampling," IEEE Trans. Antennas Propag. 60, 2309-2326 (2012). 
48. F. Viani, G. Oliveri, and A. Massa, "Compressive sensing pattern matching techniques for synthesizing planar sparse arrays," IEEE Trans. Antennas Propag. 59, 467-481 (2011).

49. G. Oliveri, P. Rocca, and A. Massa, "Reliable diagnosis of large linear arrays-Bayesian compressive sensing approach," IEEE Trans. Antennas Propag. 60, 4627-4636 (2012).
50. J. A. Tropp and S. J. Wright, "Computational methods for sparse solution of linear inverse problems," Proc. IEEE 98, 948-958 (2010).

51. O. M. Bucci and G. Franceschetti, "On the degrees of freedom of scatteredfields," IEEE Trans. Antennas Propag. 37, 918-926 (1989).

52. S. Ji, Y. Xue, and L. Carin, "Bayesian compressive sensing code," (2013), http://people.ee.duke.edu/lcarin/BCS.html. 